\title{
Adolescencias y maternidad en sectores de pobreza de Montevideo. Un estudio del vínculo temprano.
}

\section{Adolescence and motherhood in poor areas in Montevideo. An early bond case study}

\author{
Ana Cecilia Marotta Méndez \\ Universidad de la República, Uruguay \\ (Rec.: agosto de 2017 - Acept.: noviembre de 2017)
}

\begin{abstract}
Resumen
La presente investigación profundiza en el conocimiento sobre los vínculos tempranos en adolescentes de sectores de pobreza. Explora dimensiones del problema de la maternidad y embarazo adolescente no intencional, escasamente estudiados, al mismo tiempo que busca contribuir al desarrollo de políticas de prevención en salud para este sector de la población. Se realizó un estudio de tipo exploratorio y descriptivo en base a un abordaje cualitativo utilizando entrevistas en profundidad y observación del vínculo madre-hijo/a. Los resultados muestran que en las interacciones tempranas se intensifica el conflicto entre las necesidades propias de las adolescentes y las de sus hijos. El amamantamiento es la actividad más frecuente en la interacción y se constituye en una respuesta para necesidades secundarias a la nutrición. Se encontró que la madre de la adolescente es una figura clave para la red de sostén, así como la presencia del padre de los niños. Se discuten los hallazgos poniendo el énfasis en aquellos aspectos que resignifican la perspectiva teórica sobre vínculo temprano, a la luz de las condiciones materiales y subjetivas de las adolescentes.
\end{abstract}

Palabras claves: adolescencias, maternidad, vínculo temprano

\begin{abstract}
The present investigation deepens in the knowledge on the early bonds in adolescents of poverty sectors. It explores dimensions of the problem of maternity and unintentional adolescent pregnancy, scarcely studied, at the same time that it seeks to contribute to the development of health prevention policies for this sector of the population. An exploratory and descriptive study was carried out based on a qualitative approach using in-depth interviews and observation of the mother-child bond. The results show that in the early interactions the conflict between the needs of adolescents and their children intensifies. Breastfeeding is the most frequent activity in the interaction and constitutes a response for secondary needs to nutrition. It was found that the mother of the adolescent is a key figure for the support network, as well as the presence of the father of the children. The findings are discussed with an emphasis on those aspects that resignify the theoretical perspective on early bonding, in light of the material and subjective conditions of adolescents.
\end{abstract}

Keywords: adolescence, motherhood, early bond 


\section{Introducción}

La presente investigación tiene como objetivo profundizar en una línea de análisis referente al vínculo madre-hijo. Se entiende que profundizar en el conocimiento sobre los vínculos tempranos en adolescentes de sectores de pobreza permite estudiar aspectos del problema de la maternidad y embarazo adolescente, escasamente explorados, al mismo tiempo que contribuye a la definición de políticas de prevención en salud. El presente estudio tuvo entre sus objetivos conocer las características de las interacciones tempranas, explorar las redes de sostén del vínculo madre-hijo y las significaciones de maternidad. Se buscó indagar sobre algunos aspectos de sus itinerarios y trayectorias previas al nacimiento de sus hijos, así como las condiciones materiales concretas donde los vínculos tempranos acontecen.

Se utilizó una metodología cualitativa, empleando la entrevista en profundidad y la observación participante. El estudio articula el análisis de las narrativas recogidas en el trabajo de campo con la observación de las interacciones de las adolescentes con sus hijos, permitiendo comprender aspectos de la constitución subjetiva de ambos integrantes de la díada y de las prácticas que se producen en su entorno familiar inmediato.

Los resultados ponen en discusión las teorías del vínculo temprano que focalizan la díada madre-hijo en detrimento de la figura del varón adolescente para el sostenimiento del vínculo. También se jerarquizan las condiciones sociales de producción de los vínculos, ya que en ellas residen en buena parte, las posibilidades de nuevas marcas en la subjetividad de las adolescentes. Por último, se entiende que no es posible abordar el vínculo temprano sin considerar el lugar simbólico que tiene su hijo al darle una identidad propia: la de ser madre.

El trabajo se organiza en cinco apartados. Luego de la introducción se presenta el problema del embarazo en adolescentes en Uruguay y una revisión de antecedentes de investigación en el contexto nacional. Posteriormente, se presentan las consideraciones teóricas desde las cuales partió el estudio. El siguiente apartado, da cuenta de la metodología de investigación, técnicas y procedimientos utilizados. Finalmente, se presentan los resultados y seguidamente la discusión sobre la información recogida, incluyendo nueva literatura que permite arribar a conclusiones finales.

La fecundidad adolescente de la población uruguaya se mantiene elevada en comparación con el promedio de América Latina y con otras regiones del mundo (López \& Varela, 2016), siendo relevante su desigual distribución, al concentrarse en sectores con necesidades básicas insatisfechas y bajos niveles educativos. Así lo advierte el Atlas sociodemográfico y de la desigualdad social en Uruguay (Varela, Pardo, Lara, Nathan \& Tenenbaum, 2014), marcando la correlación que existe entre fecundidad y carencias críticas. Por otro lado, persisten los procesos sociodemográficos que ya habían dejado en evidencia diferencias en la reproducción biológica de la población uruguaya según su estratificación social y la existencia de dos modelos reproductivos (Paredes, 2003; Paredes \& Varela, 2001). Por un lado, los sectores más favorecidos adoptan un comportamiento equiparable al de las sociedades desarrolladas, retrasando la edad en que las mujeres realizan su tránsito hacia la maternidad, y por otro lado, una fecundidad adoles- cente alta en sectores desfavorecidos.

Si bien la fecundidad de las mujeres se redujo entre 1996 y 2011, no deja de ser un problema significativo desde el punto de vista social y de la salud, ya que las cifras se traducen en un contingente de niños, niñas y adolescentes que no cuentan con condiciones adecuadas para su desarrollo. Existen diversos trabajos que vienen desarrollando investigaciones y aportes a los temas vinculados con pobreza infantil (Cerutti, Canetti, Duarte \& Parafita, 2014; Roba, 2013), remarcando la importancia de intervenir en las etapas tempranas de la vida. En particular, en el estudio del Grupo Interdisciplinario de Estudios Psicosociales (1996) se plantean hallazgos muy elocuentes acerca de cómo se manifiesta la pobreza en el desarrollo de niños de 2 a 5 años en familias pobres del Uruguay. Destacan en sus conclusiones, que estos niños tienen mayores índices de retrasos que sus pares de medios socioeconómicos más favorecidos, en el desarrollo del lenguaje y del pensamiento simbólico. Esto se proyecta posteriormente en el fracaso escolar y en las dificultades de integración social.

Los estudios en Uruguay, durante la década de los años noventa, se centraron en las características socio demográficas de los hogares, la discusión acerca de la definición del problema embarazo adolescente y los factores de riesgo y de protección (Correas, 1996; Guemberena, 1989; Oberti, 2002; Pons, 1991). Díaz Rosselló, Guerra, Strauch, Rodríguez y Bernardi (1991) plantearon que la variable que corresponde al medio de origen es más importante que la edad para valorar el riesgo de la situación en sí. También destacan un funcionamiento matriarcal de las familias pobres, con un lugar paterno excluido y donde se hipervalora el lugar de la mujer como madre prescindiendo de otros soportes psicosociales. Se destacan los estudios del Centro Latinoamericano de Perinatología (CLAP-OPS) de la Organización Panamericana de la Salud, que indagan acerca de las motivaciones para el embarazo, centradas en el valor y estatus que ofrece la maternidad cuando se cercenan otras posibilidades de gratificación social y asunción de roles adultos. Como factores protectores mencionan la existencia de familia y de pareja, y como factores de riesgo el bajo nivel educativo y la exclusión del mercado laboral (Guemberena, 1989). Por otra parte, el estudio titulado "Uruguay adolescente, maternidad adolescente y reproducción intergeneracional de la pobreza" de Laurnaga (1995) estableció algunas líneas de interpretación sobre las motivaciones del embarazo en adolescentes, como vía de crecimiento y de ingreso al mundo adulto, como acto de reconocimiento y revaloración por parte de sus familias y medio social, como regresión a la relación infantil con su propia madre, entre otros. A inicios del siglo XXI, las investigaciones incorporan una perspectiva de género así como un enfoque en salud sexual y reproductiva. Remarcan la necesidad de que este enfoque adquiera mayor relevancia en las prácticas de los operadores del sistema de atención a la salud (López, Benia, Contera \& Guida, 2003). En esta línea, el estudio de Amorín, Carril y Varela (2006) explora los aspectos subjetivos y representaciones sociales que los/as adolescentes construyen en relación a la maternidad y a la paternidad, planteando algunas interrogantes acerca de los procesos de producción de subjetividades. Entre las cuestiones a profundizar señalan los aspectos placenteros de la maternidad y la distancia entre los discursos y las prácticas. Los investigadores insisten en que las dificultades en el ejercicio de la paternidad y maternidad en los/as adolescentes están vinculadas, entre otros factores, a la "ne- 
cesidad de resignar la posición subjetiva de hijo para asumir la nueva condición de madre o padre" y que "esto supone un proceso de reorganización psíquica" (Amorín et al., 2006, p. 193). El trabajo de reciente publicación realizado sobre maternidades adolescentes y desigualdad social (López \& Varela, 2016) continúa esta línea de investigación, buscando indagar sobre los "núcleos duros" que generan la perpetuación de los mayores índices de fecundidad adolescente en sectores de pobreza. Los resultados les permiten afirmar que el embarazo no intencional en adolescentes es producto de la desigualdad social y por este motivo, debe tomarse como problema social y objeto de política pública.

En otro orden, los estudios sobre vínculo temprano a nivel nacional son profusos aunque tienen ya varios años, destacándose los aportes de Freire de Garbarino (1992), Altmann et al. (2001), Díaz Rosselló et al. (1991). Estos trabajos abrieron un nuevo campo de conocimientos en nuestro país en el ámbito de la denominada salud mental y de la primera infancia. Sin embargo, se han concentrado en el vínculo materno filial, naturalizando el lugar de la mujer como figura principal para los cuidados de los hijos.

La adolescencia es una etapa evolutiva, descrita desde la Psicología, para denominar un período de la vida entre la infancia y la adultez. Es un constructo teórico que está relacionado con el pasaje de la familia medieval a la familia moderna, y con el surgimiento de la infancia. Los cambios en la sociabilidad y el lazo familiar que advienen con el surgimiento de la familia moderna van instalando la lógica de la familia como espacio privado y terreno para el desarrollo de la mujer (Singly, 2007), a contraposición del espacio público como patrimonio del hombre, paters-familia. Ariés (1981) puntualiza cómo en el siglo XVIII los niños eran criados en otras familias a la biológica o inclusive por nodrizas, siendo que a fines de este siglo se modifica la imagen de la madre. Este cambio resulta particularmente interesante ya que se instala por un lado la idea del niño como objeto de cuidados y se desarrolla la idea del instinto maternal (Badinter, 1984) que sostiene el mandato social para las mujeres. Según esta idea, las mujeres por su sola condición de tales, desearán ser madres como un fenómeno natural mediado por la aparición del instinto, y no como un fenómeno de la cultura. Según este mito, la confirmación del ser femenino pasaría por el ser madre incluyendo: la sacralización de la maternidad, la noción de instinto maternal, la noción de deseo de hijo (Fernández, 1994). Veremos a continuación algunas consideraciones acerca de adolescencias y maternidades con las cuales partió la investigación, así como también la perspectiva teórica sobre el concepto de vínculo y vínculo temprano.

La adolescencia es un período de alta complejidad psíquica y crisis, signada por importantes pérdidas para el sujeto: de su cuerpo, de su identidad de niño y de sus padres ideales (Aberastury \& Knobel, 1972). Susana Quiroga (1997) menciona las transformaciones en el cuerpo, el hallazgo del objeto exogámico, el cambio en los vínculos familiares, con grupos e instituciones, la decisión vocacional como aspectos de esta etapa vital. La pubertad -entendida en el tránsito de la infancia a la adolescencia- pone de manifiesto niveles de transformación corporal que van más rápido que los procesos psicológicos que acompañan el crecimiento. Desde el punto de vista psíquico, el púber se ve forzado a producir nuevas significaciones acerca de su identidad, siendo un período de búsqueda identitaria y de experimentación, abriéndose a la circulación por espacios más allá de la familia, como por ejemplo, el grupo de pares, la escuela, el liceo, clubes u otros lugares como pueden ser la propia calle, los bailes, entre otros. Es así como las formas de transitar la pubertad y las adolescencias se singularizan en relación al contexto socio-histórico por el que transitan.

En el estudio diferenciamos "embarazo adolescente" de "maternidad adolescente", ya que esta última hace a un hecho cultural más que biológico. Como dice Giberti (1997), por maternidad "entendemos la crianza, la tutela y los estímulos afectivos de la prole. La reproducción es un aspecto biológico de la misma" (p.141). En relación a cómo la experiencia de maternidad se singulariza en los contextos de pobreza, se plantea que la ecuación simbólica mujer=madre es la que prevalece en la subjetividad de las adolescentes pobres. Para el caso de las madres pobres señalamos la operatoria de una doble violencia: por un lado existe una violencia económica-social que vulnera sus derechos y las empuja a la exclusión social y por otra parte, una violencia simbólica de género (embarazo no deseado) dada por la imposibilidad de interrumpir el embarazo, la dificultad de encontrar trabajo, la responsabilidad casi absoluta por el cuidado de la progenie, etc.

El Psicoanálisis de las configuraciones vinculares, establece que el acontecimiento de tener un hijo inaugura una nueva situación para el psiquismo, produciendo nuevas marcas en la subjetividad. ¿Cómo se producen estas nuevas marcas en la subjetividad para el caso de las adolescentes madres? Según René Kaës (2002), para que un vínculo se establezca, algunas partes psíquicas de cada sujeto deben ser abandonadas para ser investidas en el vínculo. Procedemos de una ligazón y lo prematuro del nacimiento biológico muestra el desamparo inicial y nuestra dependencia al objeto. Esta unidad de ser en la relación con el otro es básica para que haya sujeto, y en este contexto, entendemos vínculo como la "construcción de un espacio psíquico dotado de una realidad propia, común y compartida por dos o más sujetos" (Kaës, 2002, p. 7).

El interés y los desarrollos teóricos en relación al vínculo madre-hijo son profusos y muy ricos. A partir de Winnicott (1979, 1993) varios autores han hecho aportes a este concepto, tales como Bowlby (1990), Brazelton y Cramer (1982), Cramer (1998), Kreisler, Soulé y Fain (1974), Lamour y Lebovici (1989), Lebovici (1983), Stern (1991, 1997, 1999). Asimismo, la observación de lactantes con la técnica de Esther Bick y los desarrollos de Selma Fraiberg (1980), han ampliado la perspectiva psicoanalítica sobre el vínculo madre-hijo. Estos desarrollos se focalizan en las competencias, creatividad y búsqueda activa del bebé, destacándose las conductas y el repertorio de este último para interactuar con la madre. Stern (1997) se involucra no sólo en el mundo del bebé sino que además se detiene en el proceso de subjetivación que atraviesa la mujer al dar a luz y hacerse cargo de la crianza. Define la "constelación maternal" como "una nueva y única organización psíquica (...), que determinará un nuevo conjunto de tendencias a la acción, sensibilidades, fantasías, temores y deseos, (...) principal eje organizador de la vida psíquica de la madre" (Stern, 1997, p. 209). 


\section{Método}

Se implementó un estudio transversal, de tipo exploratorio y descriptivo en base a una metodología cualitativa.

Se definió una muestra de mujeres adolescentes, pertenecientes a sectores de pobreza, entre 10 y 18 años de edad, residentes en Montevideo, que hubieran cursado la experiencia del embarazo y parto, y que estuvieran a cargo de la crianza de sus hijos entre 0 y 24 meses. Se tomó esta cohorte de edad siguiendo los criterios establecidos por la Organización Mundial de la Salud [OMS] en cuanto a las edades del período de la adolescencia (OMS, 1990). El límite de 2 años en la edad de sus hijos se estableció para identificar las características del vínculo temprano. Se excluyeron adolescentes con discapacidad psíquica y/o intelectual. Se efectuó un muestreo intencional teórico que tuvo en cuenta el criterio de saturación de la información (Glaser \& Strauss, 1967).

La captación se realizó a través de organizaciones gubernamentales y no gubernamentales del sector salud, que gestionan proyectos a nivel territorial que atienden población de sectores de pobreza. Los casos fueron incluidos tomando en cuenta la heterogeneidad de las edades de las adolescentes y sus hijos, y la accesibilidad estuvo dada por la disposición de los equipos que gestionan los servicios para dar viabilidad a la propuesta de investigación.

Todas las adolescentes participaron de forma voluntaria, previa firma del asentimiento y la solicitud de firma del consentimiento de sus madres u otro adulto referente. Se explicitó que se mantendría la confidencialidad de la información aportada y que se tomarían los recaudos necesarios para proteger el anonimato de las participantes del estudio. Asimismo, se solicitó autorización para grabar las entrevistas y para filmar las situaciones observadas.

Se realizaron ocho entrevistas en profundidad y siete observaciones del vínculo madre-hijo, siendo una de las observaciones fallida ya que si bien la misma fue acordada, luego la adolescente no concurrió ni tampoco respondió a los nuevos intentos para concretarla.

A partir del uso de estas técnicas combinadas se buscó acceder a las narrativas de las adolescentes tanto a través del discurso como de sus prácticas. Las entrevistas se realizaron en locales brindados por los servicios (policlínica, centro de salud, local de la ONG), y las observaciones tuvieron lugar en el domicilio de las adolescentes o en los espacios de la vida cotidiana que ellas escogieron. En dos casos se eligieron lugares alternativos que fueron la casa de la abuela paterna de la niña y una plaza pública. En este último caso la observación no se concretó.

Se elaboró una ficha individual con datos sociodemográficos básicos que luego fueron sistematizados.

La entrevista en profundidad se realizó en base a una pauta semi-estructurada, que permitió indagar distintos aspectos del vínculo de las adolescentes con sus hijos. Las entrevistas fueron grabadas, luego transcritas, revisadas y corregidas. En el caso de las observaciones se contó con el adiestramiento previo en la técnica de observación de lactantes de Esther Bick (1964), que pone el acento en que el observador desarro- lle al máximo la capacidad de receptividad, distinguiendo un nivel perceptivo, un nivel empático y emocional y una receptividad inconsciente. La consigna inicial en las observaciones fue "quiero conocer y observar cómo te relacionas con tus hijos, en tu medio cotidiano tratando que sea lo más natural posible". La posición de la investigadora fue la del observador participante, optando por un tipo de participación moderada (Spradley, 1980) incluyendo la experiencia del observador como fuente de obtención de datos para la comprensión de los fenómenos observados (Houzel, 1999). Para dichas observaciones se realizaron notas de campo "condensadas" (Spradley, 1980) tomadas inmediatamente después del trabajo de campo.

Toda la información recogida se analizó relacionando los datos de entrevistas con el material de las observaciones. Se hizo una lectura caso a caso para luego realizar un análisis transversal entre los mismos. A partir de lecturas sucesivas y del marco conceptual se construyeron las categorías para el análisis. Se incluyeron categorías emergentes que no estaban contempladas inicialmente en el marco teórico de la investigación. Los resultados se agruparon en cuatro dimensiones: I.- Significados de la maternidad. II.- Los primeros vínculos de la vida III.- El lugar del varón: significados y prácticas. IV.- Los textos de la pobreza. En el artículo se presentan los que refieren a la segunda dimensión con el fin de focalizar en este punto.

\section{Resultados}

Se presenta en primer lugar el perfil de las participantes (Tabla 1).

Tabla 1. Edad de las adolescentes, edad y sexo de los hijos

\begin{tabular}{cc}
\hline $\begin{array}{c}\text { Edad de la } \\
\text { adolescente }\end{array}$ & Edad y sexo del hijo \\
\hline 14 años & Varón de 4 meses. \\
17 años & $\begin{array}{c}\text { Varón de } 1 \text { año } 9 \text { meses y beba } \\
\text { de } 4 \text { meses y medio. }\end{array}$ \\
18 años & Mujer de 1 año 7 meses. \\
16 años & Varón de 1 año. \\
18 años & Varón de 2 años. \\
15 años & Mujer de 9 meses. \\
17 años & Mujer de 2 años y beba de 5 \\
meses.
\end{tabular}

Todas habían completado el ciclo escolar pero una vez en el liceo, no pudieron culminar el primer año. A la fecha de sus embarazos estaban incluidas en el sistema formal ya sea terminando la escuela o cursando el liceo. Sólo en un caso el abandono del liceo fue previo al embarazo.

Casi la totalidad de las participantes tenían cobertura en salud en servicios del sector público con excepción de una de ellas que disponía de cobertura privada mutual. 
En relación a la integración del núcleo de convivencia, siete de las adolescentes viven con su madre y en un solo caso con su pareja e hijos exclusivamente. Dos de ellas convivían con sus madres y la pareja de las madres. En el resto de los casos, las parejas vivían en sus respectivos hogares.

Las edades en que cursaron sus embarazos, osciló entre 13 y 15 años. Más de la mitad, con 15 años había tenido su primer hijo.

A partir de las entrevistas fuimos indagando en las trayectorias de las adolescentes, así como las observaciones nos permitieron transitar por sus barrios e ingresar a la cotidianeidad de sus vidas.

En relación a sus embarazos, en ninguno de los casos este fue producto de una relación ocasional o de progenitor desconocido. Por el contrario, las siguientes viñetas muestran los diferentes tipos de vínculo que las entrevistadas mantenían con el progenitor al momento de la concepción:

"Yo a D lo conocí porque era hermano de mi cuñada (...) Era el hermano de la señora de mi hermano. (...) Entonces... cada vez que iba de visita lo conocía. Ta', lo veía cada vez que iba. Y cuando me fui a vivir con mi hermano... Que yo viví con él cuando tenía catorce, trece por ahí, y tá. Y me arreglé con D, estábamos de novios y al poquito tiempo quedé embarazada" (Loana, 17 años).

"(...) nosotros estábamos bien. Nos llevábamos bien, íbamos para todos lados juntos. Estábamos todo el tiempo juntos. $Y$ después tá, cuando ya sabíamos los dos que yo estaba embarazada, como que nos empezamos a llevar más".

(Carolina, 15 años).

En la mayoría de los casos las parejas se mantuvieron luego del parto y al momento de la investigación. Algunas entrevistadas, plantearon que el embarazo fue planificado junto a la pareja, sin embargo, las respuestas mostraron cierta ambivalencia, así como los modos de negociación con el progenitor, dejando en evidencia la manera en que pesó el deseo del varón en la decisión:

"Sí, nosotros al principio nos cuidábamos pero después los dos queríamos tener un hijo. Y yo le decía noo, más adelante. $Y$ después me convencí, yo también quería tener un hijo (...) Y tá" (Sabrina, 16 años).

"No, porque mirá (...) yo lo había conocido a él y viste cuando te enamorás así y querés tener un hijo y no pensás. Yo quería tener un hijo y después me puse a pensar para qué voy a tener un hijo, soy muy chica y voy a tener toda la vida por delante. ¡Y ahí me enteré que estaba embarazada! (...) Yo le dije que yo no quería tener un hijo, pero no sé tá (...) ¿Y él? (...) Nada, iel sí!" (Josefina, 15 años).

El embarazo fue vivenciado como un hecho ajeno y que se impone. Frente al mismo, algunas entrevistadas expresaron su ambivalencia y las peripecias internas que atravesaron a partir de la noticia y durante el transcurso del mismo.

"Porque yo estaba embarazada, pero yo quería estar embarazada pero por momentos no quería. Quería sacarme la panza y después ponérmela otra vez (...) Sacarme la panza por ejemplo, y ponérmela otra vez una cosa así, por momentos no quería" (Lucía, 16 años).

"Quedé embarazada (...) Yo no quería (...) Bueno no quería (...) ¡no quería tener un hijo viste! Porque era muy chica, pero tá, lo tuve" (Josefina, 14 años).

Por otro lado, mencionaron hechos traumáticos previos al embarazo, propios o de sus parejas: la muerte de uno de los padres, períodos de ingesta de fármacos o consumo de sustancias, la salida forzosa del lugar donde vivían, la muerte de otros bebés, violencia doméstica:

"(...) mi papá falleció y mi madre estaba embarazada de 3 meses (...) yo que sé, en ese momento sentí toda la atención hacia ella. Yo me quería reflejar en otros lados y como que no podía, todo se me derrumbaba. Y bueno, a raíz de eso surgieron varios temas (...) ese año, para cumplir los 14 conocí al papá de mi nena (...) y ahí como que encontré un afecto que más que como un novio, un marido o como le quieras llamar, era como mi compañero, alguien que me enseñaba, que me ayudaba a salir de donde yo estaba. Ah, él después tuvo problemas, cayó preso. O sea, yo quedé embarazada, cuando él cayó preso, yo quedé embarazada" (Camila, 17 años).

"Porque yo quería un bebé y criaba al bebé de mi tía. Más bien fue por eso, pero también porque yo quería un hijo. $Y$ tá, lo tuvieron en el Pereyra Rosell y lo mataron ellos. Con los pulmones (...) Sí, porque como mi tía trabajaba todo el día prácticamente lo cuidaba yo. Al bebé lo cuidaba yo"

(Sabrina, 16 años).

Los relatos permiten observar que en muchos casos, para las adolescentes tener un hijo ha significado una salida a situaciones traumáticas o una manera de llenar vacíos afectivos.

Sobre el uso de métodos anticonceptivos la mayoría de las entrevistadas mencionaron haberlos usado, pero restando importancia a los mismos y en algunos casos con escasa información o dificultades en su comprensión, lo que pone en evidencia la escasa o nula apropiación de sus derechos sexuales y reproductivos. El ejercicio de la sexualidad quedó así, ligado a la reproducción y en la mayoría de los casos, no fueron embarazos planificados. En este sentido, estamos frente a embarazos no intencionales, es decir aquellos embarazos que tienen lugar en un momento no esperado para la persona o que no fueron buscados.

\section{"Yo desde el primer momento que tuve relaciones con el papá de ella no me había cuidado" \\ "Porque no me cuidaba y tá (...) No, no quería tener un hijo pero no sabía, no me cuidaba y tá" (Emilia, 18 años)}

Desde antes del nacimiento del hijo/a el vínculo es conflictivo y ambivalente, y se expresa a través de sus cuerpos durante el transcurso del embarazo. Embarazos caracterizados por amenazas de aborto espontáneo y de parto prematuro, las adolescentes reaccionaron con rechazo al alimento o ingesta en grandes cantidades, dificultades para manejar la ansiedad, etc. Si bien no fue objetivo de investigación indagar sobre la interrupción del embarazo, el tema surgió espontáneamente en los relatos de las adolescentes. Transcribimos dos viñetas donde se expresaron abiertamente sobre la eventualidad del aborto: 


\section{"(...) Ta, yo me lo quería sacar pero tá" "¿Lo hablaste con D?"}

"Sí, lo hablé. Pero no sabíamos que hacer. Yo decía 'me lo quiero sacar' pero no hacíamos nada, porque no sabíamos nada que hacer. Ta, después tá, cuando yo fui al médico, que esperé cómo dos meses, estaba de tres meses cuando fui al médico. ¡Sacármelo de tres meses! entonces tá (...) un señor así, que sabía de esas cosas, me dijo, pero tenía que hacer muchas cosas (...) Sí, que tenía que dejar de comer una semana y tomar unos tés. Que me podía venir hemorragia, todas esas cosas entonces yo decía no (...) decía 'Tenés que ver que te puede pasar a vos algo, tenés que pensar por el otro bebé.' Muchas cosas, entonces me dio miedo que pasara algo y tá. Seguí" (Loana, 17 años).

"Si, mi mamá me dijo por mi bien, que si yo no lo deseaba, no lo quería, ella me daba la plata para que yo no lo tuviera. O sea, no me lo dijo obligándome ni nada, ella me dijo por mi bien que yo era chica y capaz no podés o no querés. $O$ en este momento no estás preparada. Yo en ese momento, o sea, le dije que no, que yo lo quería" (Camila, 17 años).

La idea de la interrupción del embarazo aparece en la mayoría de los casos ligada al camino que le ofrece su propia madre, pero no logra efectivizarse sino que el embarazo continúa y es vivido como un proceso que "le" sucede a sus cuerpos, así como el parto. La decisión y la posibilidad de interrumpir el embarazo en el caso de las adolescentes que desearon hacerlo, estuvo en estrecha relación con los recursos disponibles: información confiable y accesible, recursos afectivos y económicos. En el momento en que se realizó la investigación, el aborto era considerado delito en Uruguay, cuestión que fue modificada con la aprobación en el año 2012 de la Ley No. 18987 de interrupción voluntaria del embarazo. Si bien esto marcó un límite para que se pudiera abordar desde el sistema de salud, en el desarrollo de las entrevistas no estuvo presente como limitación.

A partir del nacimiento se modificó sustancialmente el vínculo madre-hijo. En todos los casos los primeros meses e interacciones fueron vividos con mucha exigencia y agobio por la situación de hacerse cargo de su bebé.

"iAh! yo me sentía mal ya de por sí. Y quedé media boleada viste, cansada pero ¡cansada! Te cansás, tenía sueño y tá, no sabía hacer las cosas. Tenía miedo, a veces tenía miedo de dejarlo llorar mucho. Porque no le daba la teta porque me dolía estaba media... ifuah!" (Lucía, 16 años)

Manifestaron el conflicto entre sus propias necesidades y las de sus hijos. Como adolescentes, intentaron que la realidad se acomodase a sus expectativas, lo que dificultó que pudieran establecer las rutinas que sus hijos necesitaban: hambre, sueño, dolor.

"(...) pero hacía más cosas viste (...) Estaba con mis amigas, iba con mis amigas a bailar. Iba para un lado y para otro con mis amigas. Yo que sé, íbamos a bailar, ella se quedaba en mi casa yo iba para la casa de ella. Estaba con el padre de $L$, y tá. Una banda de amigos, yo que sé" (Lucía, 16 años).

"Me dice que de noche ella duerme con la teta para afuera, porque él se despierta, chupa y así está toda la noche. Que ella duerme porque al dejar la teta para afuera" (Observación: Lucía, 16 años).

Las adolescentes refirieron situaciones de tensión en el vínculo ligados a los momentos de separación y unión cuando se presenta el gateo y el acto de la alimentación:

"Cuando era chica era muy pegada a mí, cuando tenía más o menos a los 4 meses que ya me empezaba a conocer... sabía que yo era la madre. Era re re pegada como hasta los 8 meses... Yo a veces no podía hacer nada en mi casa porque ella lloraba, quería estar arriba mío. Y yo la dejaba en el piso para que ella gateara o pa' que ella hiciera otras cosas para no estar arriba mío. Pero no, ella lloraba, lloraba, lloraba. Quería estar conmigo y tá. No la podía calmar" (Emilia, 18 años).

"Me da mucho trabajo para comer. (...) Sí, para comer conmigo. Conmigo no quiere nada, con mi madre sí o cualquiera que le dé. Pero eso sí, no me puedo ir de mi casa porque... ¡Cada Ilanto!" (Sarina, 18 años).

A su vez, expresaron la dificultad para transitar el conflicto de independencia- dependencia, ya sea incentivando las conductas motoras como los cambios en la alimentación, y lo que esto implica en tanto proceso de separación y autonomía del niño. También en las observaciones, se confirmaron y verbalizaron dificultades para que el hijo pueda estar a solas, refiriendo la necesidad de la presencia del otro para calmar o apaciguar las ansiedades.

Su hijo se adelanta con el andador y por momentos se suelta pero no avanza, queda parado en un sitio. La abuela dice que a L le gusta que estemos así con él, pregunto ¿cómo?, "...que a él no le gusta estar solo", dice la madre (Observación: Lucía, 16 años)

Pregunto si llora mucho, el papá sonríe y dice que sí, mirando a la abuela. La sra. desde la cuerda, comenta que ellos le dan mucho brazo, y que la beba solo quiere brazos y que sino... llora. (Observación: Camila, 17 años).

Durante todo este período a posteriori del nacimiento, sus madres jugaron un papel clave, tornándose un factor de protección. No solamente en lo que refiere al cuidado y atención de los bebés y niños, sino para sostener y acompañar la tramitación de las angustias y desbordes afectivos de sus hijas.

Las adolescentes desestimaron los juegos sonoros o las necesidades que fueran más allá del alimento o la higiene de sus hijos. Los juegos de mayor intensidad y donde se produce una interacción más estimulante es con el padre. En la mayoría de los casos las adolescentes mantuvieron el vínculo con el progenitor de sus hijos y a su vez, estos sostuvieron el vínculo con ellos. Algunas características observadas del vínculo del padre con sus hijos, fueron el involucramiento en juegos de mayor intensidad, juegos y actividades físicas sin mediación de juguetes, más bruscos y audaces, meciendo verticalmente al hijo y teniendo más contacto táctil que visual.

"Pero conmigo mucho no lo dejo jugar. Le digo para jugar pero se va con él... Le gusta jugar a la pelea. Subir a la cama y saltar y esas cosas... a él le gusta jugar a esos juegos medio brutos" (Lucía, 17 años). 
"La adolescente se queda en la silla con actitud callada de tranquilidad y la abuela trae a la bebé a los brazos del papá. Primero la coloca sentada mirando hacia mí y enseguida la gira tomándola del tronco y subiendo y bajando a G., jugando... la baja y la vuelve a sentar, $G$ está con expresión de atención y placer" (Observación: Camila, 16 años).

En relación a la lactancia, el pecho que marcó el comienzo de un vínculo, se transformó en respuesta recurrente para calmar las ansiedades del bebé. Las observaciones realizadas, así como algunas referencias en los discursos de las adolescentes, mostraron la cualidad del vínculo que establecen con sus hijos a través del acto de amamantar.

"Sólo teta. Ahora se la pongo, pero ahora ya no me sale más leche, pero a veces cuando está arriba mío, me levanto el buzo, se la pongo así y ella chupa... pero no chupa... es un mimo y yo le doy, pero a mí me gusta darle. Pero tá, el médico me dijo que se la tenía que sacar porque no estaba engordando bien. $Y$ se la tuve que sacar... (...)Y fui al control y el médico me dijo que no tenía que dar más. Llegué a mi casa y me puse aloe, ella puso cara fea y no tomo más.... Le dije que viniera, para ver si ella volvía a querer... pero como no quería dije 'no tomás más'y no tomó más" (Emilia, 18 años).

Si bien algunas de las entrevistas mencionan lo gratificante de la experiencia de amamantamiento, la mayoría menciona dificultades que en muchos casos impiden la continuidad de la lactancia.

Por parte de las adolescentes, la iniciativa más importante en la interacción -y repetida en las observaciones-, es el pecho como respuesta principal para distintas necesidades secundarias a la nutrición: para inducir el sueño, tranquilizar, apaciguar, ser mirado, aliviar tensiones en el bebé. Las siguientes viñetas son muy claras al respecto:

Se escucha que la bebé, que se fue para el cuarto con el padre, Iloriquea o se molesta. L está sentada en la silla y su hijo dando vueltas por el comedor. En un momento sale el padre con la bebé en brazos y se la da a la adolescente. Ella la pone al pecho diciendo que ya debe de tener hambre, pregunto cada cuánto la pone al pecho, y me dice que no tiene hora, que todo el tiempo, que cuando ella pide. Que cuando la puso antes no tenía hambre, esta vez la bebé mama mientras se calma del llanto. Luego deja de tomar y $L$ vuelve a comentar que no tenía hambre. (Observación: Loana, 17 años).

Ve como se mueve A en el cochecito y me dice que el bebé tiene sueño. Pero no lo levanta hasta pasado unos minutos, A tampoco se molesta demasiado pero se lo nota inquieto. J finalmente lo levanta y le da de mamar. Me llama la atención que le dé de mamar, es más como un medio para hacerlo dormir me parece, que porque tenga hambre. Lo pone al pecho y el bebé se prende enseguida y luego de unas mamadas J dice: "se cansó". Y se va quedando dormido.

(Observación: Josefina, 14 años).

El resto de las interacciones comportamentales son escasas y el encuentro corporal entre la adolescente y sus hijos se da fundamentalmente en estas situaciones.

\section{Discusión}

Los resultados del estudio permitieron caracterizar los vínculos tempranos que las adolescentes construyen con sus hijos. La producción teórica sobre vínculo temprano se ha centrado en el binomio madre-hijo, siendo escasamente abordados otros elementos que aparecen con mucha fuerza en esta investigación y que es necesario atender para comprender la singularidad de ese vínculo: las características materiales concretas del lugar donde viven las adolescentes, el modo en que la maternidad impacta en sus vidas, la figura del adolescente varón y el papel que juegan las redes de sostén para el vínculo.

En consonancia con investigaciones en contextos similares (Castilla \& Lorenzo, 2012), las entrevistadas significan su maternidad como el proyecto vital más importante, restringiendo sus vidas al ámbito doméstico y barrial. Del discurso de las adolescentes se pudo constatar la distancia entre la idea abstracta de la maternidad como lugar ideal de existencia para la mujer, y las vivencias y relatos sobre cómo tuvo lugar el embarazo y las circunstancias que lo rodearon, así como su tránsito al ser madres. Los resultados obtenidos permiten discutir la idea que sostiene Stern (1997) sobre los procesos de reorganización psíquica, cuando hace referencia a la sintonía afectiva y la constelación maternal, ya que las adolescentes entrevistadas parecen seguir centradas en sus propias necesidades y requieren de comprensión y apoyos significativos para sostener el vínculo con sus hijos. Sostenemos que las condiciones estructurales de pobreza y las características subjetivas propias de la etapa evolutiva en la que se encuentran estas adolescentes pueden interferir en la posibilidad de empatizar y atender las necesidades afectivas de sus hijos. Los determinantes sociales a los que se enfrentan y que observamos en sus domicilios y barrios (precariedad de la vivienda, mendicidad en las familias, situaciones de calle, deserción del sistema educativo, entre otros), actúan configurando situaciones de precariedad material y de vulnerabilidad psíquica para el apuntalamiento del vínculo temprano. Los escenarios de colecho, cohabitación y/o precariedad de las viviendas, son condiciones objetivas de producción para los vínculos. También son contextos que interfieren en el desarrollo adolescente, ya que la maternidad exige el ejercicio de roles adultos, lo cual insume un costo psíquico importante para estas jóvenes, aumentando su vulnerabilidad y la de sus hijos/as.

No es posible comprender la ambivalencia emocional y el conflicto entre las necesidades propias y las de sus hijos/as, si no se realiza un análisis de las significaciones de maternidad y las posibilidades de incluir otros proyectos de vida que les permitan mayores niveles de autonomía. Siguiendo las ideas de Lagarde (2003), la maternidad se erige como dispositivo de sujeción cuando es abonado por la idea de la naturalidad, de lo instintivo y de una dimensión de la existencia que totaliza la identidad femenina. Sin embargo, cuando la maternidad está inscrita en un proyecto mayor donde la mujer dispone de un mayor repertorio de recursos y posibilidades, esta puede ser una vía de realización legítima y disfrutable para las adolescentes mujeres y no solamente la repetición de un destino.

Consideramos que no es posible producir conocimiento y/o generar prácticas de intervención (educativas, sanitarias, etc.) sobre vínculo temprano en estos sectores, sin contemplar los efectos que producen las desigualdades sociales. Es impor- 
tante tomar en cuenta, al decir de Claudia Calquín (2013), la idea de la intervención como espacio articulador de lo macro y lo micro para el trabajo con estas poblaciones.

Los resultados muestran que si bien el ejercicio maternal sigue siendo una tarea predominantemente femenina (Chodorow, 1984), es llevada adelante por otras mujeres además de la adolescente (abuelas, tías). El varón progenitor estuvo presente en el discurso como en las observaciones realizadas. Es importante destacar su presencia en tanto muestra el deseo de involucrarse más activamente en el ejercicio de las funciones parentales. Generalmente, el varón no es tomado en cuenta por las organizaciones del sector salud y/o educación, quienes la mayor parte de las veces convocan y esperan a las madres como referentes de los bebés y niños. La frecuente invisibilización del progenitor varón refuerza la mirada que focaliza el vínculo temprano madre-hijo como único determinante de la construcción de la subjetividad. La participación de los progenitores en el cuidado de los hijos/as, así como la presencia de las madres de las adolescentes, son recursos de apoyo familiar que juegan un papel clave y diferencial.

Para finalizar, señalamos que si bien los embarazos no fueron intencionales, una vez asumida la decisión de continuarlo, las adolescentes significan su maternidad como el proyecto vital más importante restringiendo sus vidas al ámbito doméstico y barrial. El lugar simbólico del hijo es brindarle una identidad propia: la de ser madre. El vínculo que estas adolescentes tienen con sus hijos es ambivalente no logrando descentrarse de su lugar de hija como posición subjetiva. Tampoco logran decodificar adecuadamente las necesidades de sus hijos, asumiendo un rol materno para el cual no están preparadas.

Los resultados del estudio contribuyen al desarrollo de una agenda de investigación sobre las dificultades que implica el ejercicio de la maternidad temprana y -en particular- el vínculo con los hijos/as en condiciones de desigualdad social. Estos aspectos deberían ser tomados en consideración en el diseño e implementación de políticas públicas del embarazo no intencional así como de apoyo a las adolescentes que realizan una transición temprana a la maternidad.

\section{Referencias}

Aberastury A, Knobel M. (1973) La adolescencia normal. Buenos Aires: Paidós.

Altmann, M., Weigensberg, A., González, E., Beatriz, M., Brovetto, E., Bonifacio, N., ... \& Vieira, M. (2001). Arrullos, ritmos y sincronías en la relación madre-bebé. Revista Iberoamericana de Psicomotricidad y Técnicas Corporales, (1), 49-62. Recuperado de https://dialnet.unirioja.es/servlet/ articulo?codigo $=3749124$

Amorín, D., Carril, E. \& Varela, C. (2006). Significados de maternidad y paternidad en adolescentes de estratos bajos y medios de Montevideo. En A. López (Coord.), Proyecto género y generaciones. Reproducción biológica y social de la población uruguaya. (pp. 125-246). Montevideo: Trilce.

Ariés, P. (1981). Da Familia Medieval a Familia Moderna. En P. Ariés (Ed.), Historia social da criança e da familia
(2 ed., pp. 154-189). Rio de Janeiro: LTC Editora S.A.

Badinter, E. (1984). ¿Existe el instinto maternal?: historia del amor maternal, siglos XVII al XX. Barcelona: Paidós.

Benedet, L. \& Ramos, V. (2009). Mujeres y varones adolescentes en situación de embarazo en los servicios de salud. Montevideo: Universidad de la República.

Bick, E. (1964). Notas sobre la observación de lactantes en la enseñanza del psicoanálisis. The International Journal of Psycho-Analysis, 45(4), 558-566. Recuperado de http://www.asociacionbick.org/pdf/ notas_observacion_lactantes_ensenanza_psicoanalisis.pdf

Bowlby, J. (1990). Vínculos afectivos: formación, desarrollo y pérdida. Madrid: Morata.

Brazelton, C. \& Cramer, B. (1982). La relación más temprana: padres, bebés y el drama del apego inicial. Buenos Aires: Paidós.

Calquin, C. (2013). De madres y de expertos: la psicología de posguerra y el disciplinamiento de los cuidados maternos. Summa Psicológica UST , 10, 119-129. doi:10.18774/summa-vol10.num1-42

Castilla, V. \& Lorenzo, G. (2012). Emociones en suspenso: maternidad y consumo de pasta base/paco en barrios marginales de Buenos Aires. Cuadernos de Antropología Social, (36), 69- 89. Recuperado de http:// www.redalyc.org/articulo.oa?id=180926074005

Cerutti, A., Cannetti, A., Duarte, D. \& Parafita, D. (2014). Políticas sociales para la infancia en el Uruguay con énfasis en las edades tempranas. Propuestas de monitoreo del bienestar infantil. Montevideo: CIIPEl-UdelaR.

Chodorow, N. (1984). Introducción. En N. Chodorow, El ejercicio de la maternidad. Psicoanálisis y Sociología de la Maternidad y Paternidad en la crianza de los Hijos (pp. 13-24). Barcelona: Gedisa.

Correas, P. (1996). Ser madre y ser hijo en el marco de la maternidad adolescente. En D. Defey, Los bebés y sus padres en situaciones difíciles (Vol. 4, pp. 13-44). Montevideo: Roca Viva.

Díaz Roselló, J., Guerra,V., Strauch, M., Rodríguez, C. \& Bernardi, R. (1991). La madre y su bebé: Primeras interacciones. Montevideo: Roca Viva.

Fernández, A. (1994) La mujer de la ilusión. Buenos Aires: Paidós.

Fraiberg, S. (Ed.). (1980). Clinical studies in infant mental health. The first year of life. New York: Basic Books, Inc. Publisher.

Freire de Garbarino, M. (Coord.). (1992). Interacción temprana. Investigación y Terapéutica breve. Montevideo: 
Roca Viva.

Glaser B, Strauss L. (1967) El descubrimiento de la teoría fundamentada: estrategias para la investigación cualitativa. New York: Aldine Publishing Company.

Grupo Interdisciplinario de Estudios Psicosociales. (1996) Cuidando el potencial del futuro. El desarrollo de niños preescolares en familias pobres del Uruguay. Montevideo: El Departamento.

Guemberena, L. (1989). La gestación en la adolescencia: hipótesis y conclusiones preliminares. Programa de Entrevistas a Adolescentes Puérperas. Montevideo: CLAP/OPS, MSP.

Houzel, D. (1999) Aplicación terapéutica de la observación de lactantes en psiquiatría infantil. Revista Internacional de observación de lactantes, 2, 75-89. Montevideo: Biblioteca de APPIA

Kaes, R. (2002). Vínculo e intersubjetividad. Conferencia no publicada dictada en el Encuentro con R. Kaes en la Asociación Uruguaya de Psicoanálisis de las Configuraciones Vinculares, Montevideo, Uruguay.

Kreisler, L., Soulé, M. \& Fain, M. (1974). El niño y su cuerpo. Buenos Aires: Amorrortu.

Lagarde y de los Ríos, Marcela (2003) Los cautiverios de las mujeres: madresposas, monjas, puntas, presas y locas. México: Universidad Nacional Autónoma de México.

Lamour, M. \& Lebovici, S. (1989). Las interacciones del bebé y sus partenaires: evaluación y modos de abordaje preventivos y terapéuticos. Revista Psychiatrie de I 'enfant, 34, 171-275.

Laurnaga, M. (1995). Uruguay adolescente. Maternidad adolescente y reproducción intergeneracional de la pobreza. Montevideo: Trilce.

Lebovici, S. (1983). El lactante, su madre y el psicoanalista. Las interacciones precoces. Buenos Aires: Amorrortu.

López, A. \& Varela, C. (2016) Maternidad en adolescentes y desigualdad social en el Uruguay. Montevideo: UNFPA

López, A., Benia, W., Contera, M. \& Guida, C. (2003) Del enfoque materno infantil al enfoque de la salud reproductiva. Tensiones, obstáculos y perspectivas. Montevideo: Rosgal.

López, A. (Coord.). (2006). Proyecto género y generaciones. Reproducción biológica y social de la población uruguaya. Montevideo: Trilce.

Oberti, P. (2002). Adolescentes embarazadas. Enfoque desde la perspectiva de las involucradas. En A. Gandolfi, Psicología de la salud: escenarios y prácticas (pp. 151-163). Montevideo: Psicolibros.
Paredes, M. (2003). Los cambios en la familia en Uruguay: ¿hacia una segunda transición demográfica? En UNICEF-UdelaR, Nuevas formas de familia. Perspectivas Nacionales e Internacionales (pp. 73-101). Montevideo: UNICEF-UdelaR.

Paredes M. \& Varela, C. (2001). Aproximación sociodemográfica al comportamiento reproductivo y familiar en el Uruguay (Documento de Trabajo No67). Montevideo: UR. FCS-UM.

Pons, J. (1991). ¿Cuán riesgoso es el embarazo en la adolescencia? En Portillo, J., Banfi, M. L., Martinez, J. \& Briozzo, T. La adolescencia (pp. 235-240). Montevideo: Banda Oriental.

Quiroga, S. (1997). Adolescencia: del goce orgánico al hallazgo de objeto. Buenos Aires: Oficina de Publicaciones de la UBA.

Roba, O. (2013). Medición de la pobreza infantil en Uruguay (Documento de Trabajo 1). Montevideo: Centro Interdisciplinario de Infancia y Pobreza/ Espacio Interdisciplinario-UdelaR.

Singly de, F: (2007). Sociologia da familia contemporânea. Río de Janeiro: FGV Editora.

Spradley JP. (1980) Observación participante. Orlando: Harcourt Brace.

Stern, D. (1991). Diario de un bebé. Barcelona: Ediciones B.

Stern, D. (1999). El nacimiento de una madre. Barcelona: Paidós.

Stern, D. (1997). La constelación maternal. Barcelona: Paidós.

Varela, C., Pardo, I., Lara, C., Nathan, M. \& Tenenbaum, M. (2014). Atlas sociodemográfico y de la desigualdad en el Uruguay. Fecundidad en el Uruguay (19962011): desigualdad y diferencias en el comportamiento reproductivo. Montevideo: Editorial Trilce.

Winnicott, D. (1993). La capacidad para estar a solas. En D.

Winnicott, Los procesos de maduración y el ambiente facilitador (pp. 36-46). Buenos Aires: Paidós.

Winnicott D. (1979). Escritos de pediatría y psicoanálisis. Barcelona: Laia. 